“C 2017 IEEE. Personal use of this material is permitted. Permission from IEEE must be obtained for all other uses, in any current or future media, including reprinting/republishing this material for advertising or promotional purposes, creating new collective works, for resale or redistribution to servers or lists, or reuse of any copyrighted component of this work in other works." 


\title{
A wideband dipole antenna based on a non-uniformly segmented structure
}

\author{
Haihan Sun ${ }^{1}$, Can Ding ${ }^{1}$, Y. Jay Guo ${ }^{1}$, Raj Mittra ${ }^{2}$ \\ ${ }^{1}$ Global Big Data Technologies Centre, University of Technology Sydney (UTS), Australia, Haihan.Sun@student.uts.edu.au \\ ${ }^{2}$ EMC Laboratory, The Pennsylvania State University, USA
}

\begin{abstract}
The design of a wideband, vertically polarized omnidirectional dipole antenna based on a non-uniformly segmented structure is presented. The proposed non-uniformly segmented configuration mitigates the phase change of the currents flowing in the long dipole arms, so that the reverse currents which deteriorate the radiation pattern can be avoided, and a stable omnidirectional pattern can be maintained over a wide frequency band. Simulation results show that the proposed dipole has a wide pattern bandwidth of $66 \%$, ranging from $1.96 \mathrm{GHz}$ to $3.90 \mathrm{GHz}$, with $S_{11}$ less than $-10 \mathrm{~dB}$. Within the band, the main lobe of the dipole remains in the broadside direction in the E-plane and the antenna achieves the desirable omnidirectional radiation pattern, with a gain flatness of less than $0.3 \mathrm{~dB}$ in the H-plane.
\end{abstract}

Index Terms - wideband, vertically polarized, dipole antennas, non-uniformly segmented structure, omnidirectional pattern.

\section{INTRODUCTION}

Antennas that can provide vertically polarized omnidirectional radiation are in demand in many applications, such as wireless sensor networks (WSN), cellular networks, and wireless local area networks (WLAN) [1], in which a wide bandwidth and a high gain are usually preferred. The dipole antenna is one of the most frequently used radiators to obtain the aforementioned radiation characteristics and has the advantages of simplicity and low cost [2]. Although a large number of methods have been proposed to improve the gain and bandwidth of dipole antennas [3]-[6], the performance of the dipole has been found to be intrinsically limited by its physical size [7]. In order to simultaneously obtain a higher gain and a wider bandwidth, the size of the dipole needs to be increased. However, in traditional dipole configurations, reverse currents flow in the dipole arms when the physical length of the dipole exceeds a wavelength, and this in turn deteriorates the radiation pattern owing to the splitting of the main lobe.

The composite right/left-handed (CRLH) structure can be used to prevent the reverse currents on a long dipole, because of its capability to achieve a zero propagation constant at the zeroth-order resonant frequency [2]. The segmented line structure, as one of the CRLH structures, has attracted considerable attention, since it is relatively easy to fabricate [8]-[11]. The phase variation along this structure can be controlled by tuning the lengths and widths of the segmented strips. A dipole antenna based on a uniform segmented structure has been presented in [12], and a stable omnidirectional pattern within a wide bandwidth has been achieved.

In this paper, a novel design of the dipole antenna is proposed, based on the use of the non-uniformly segmented line structure, to achieve a wider bandwidth with high gain. The transmission characteristics of the proposed nonuniformly segmented structure are investigated. Simulation results of the impedance bandwidth and radiation characteristics of the above dipole are presented and discussed.

\section{ANTENNA CONFIGURATION}

Fig. 1 shows the configuration of the proposed wideband dipole antenna. Each arm of the dipole consists of two groups of metalized strips printed on different sides of the substrate. The strips in each group are evenly spaced but have different lengths. Two coupled microstrip lines with length $l_{i}$ and width $w_{i}$ are printed on different sides of the substrate to match the antenna. The substrate employed in this work has a dielectric constant of 2.65 and a thickness of $0.8 \mathrm{~mm}$. The dimensions of the antenna are optimized to maintain unidirectional currents flowing in the dipole arms to provide the widest pattern bandwidth. These dimensions are listed in Table I.

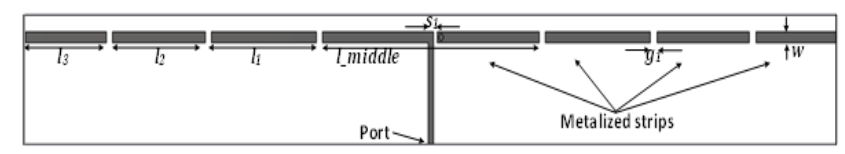

(a)

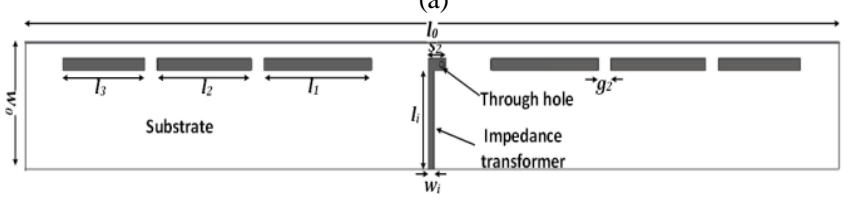

(b)

Fig. 1. Antenna configuration. (a) front view. (b) back view.

TABLE I. DIMENSION OF THE PROPOSED ANTENNA

\begin{tabular}{|c|c|c|c|}
\hline Parameter & Value (mm) & Parameter & Value (mm) \\
\hline$l_{-}$middle & 35 & $g_{1}$ & 1 \\
\hline$l_{1}$ & 17 & $g_{2}$ & 2 \\
\hline$l_{2}$ & 15 & $s_{I}$ & 0.6 \\
\hline$l_{3}$ & 13 & $s_{2}$ & 2.7 \\
\hline$w$ & 1.4 & $l_{0}$ & 131 \\
\hline$w_{i}$ & 0.9 & $w_{0}$ & 16 \\
\hline$l_{i}$ & 12.5 & & \\
\hline
\end{tabular}


To demonstrate the ability of the proposed structure to mitigate the phase change of the currents flowing in it, the current phase variations along one of the dipole arms are monitored at certain frequency points $(2,3$, and $4 \mathrm{GHz})$. The results are compared in Fig. 2 with those for a continuous strip used in conventional dipoles, as well as with those for the uniformly segmented structure proposed in [12]. The figure shows that the phase of the currents on the proposed non-uniform structure changes significantly slower than those on the uniform structure and the continuous strip, especially at $3.0 \mathrm{GHz}$. This, in turn, helps to circumvent the unwanted phase reversion problem on a long dipole, which is approximately $1.3 \lambda_{0}$ long at $3.0 \mathrm{GHz}$, and the antenna maintains a single main lobe in the broadside direction across a very wide band.

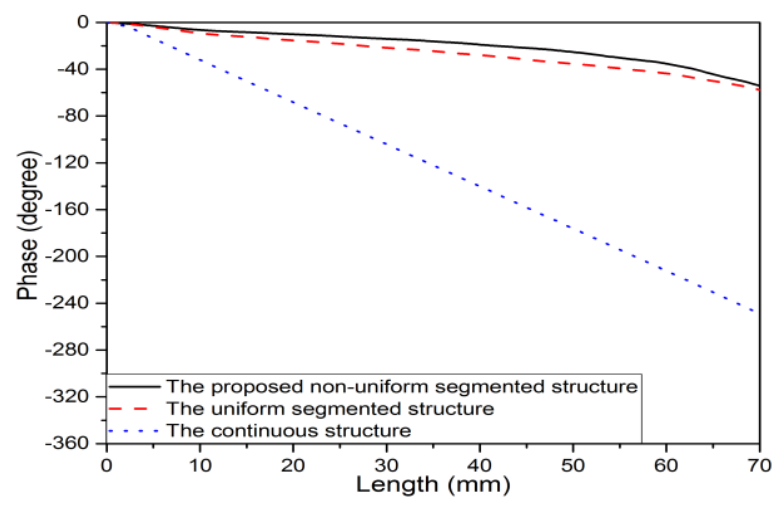

(a)

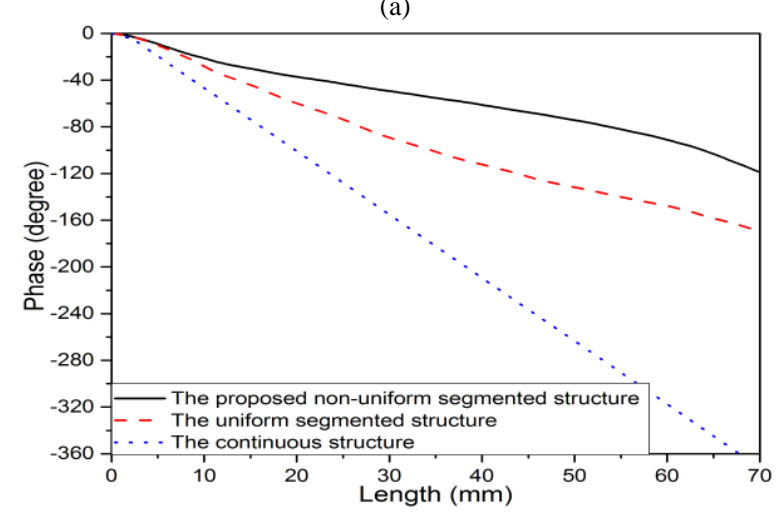

(b)

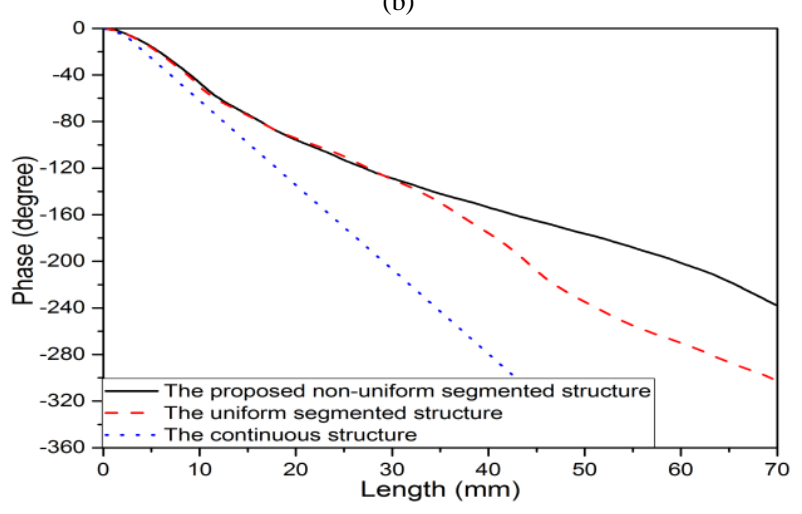

(c)

Fig. 2. Phase of current flowing in three different structures. (a) $2.0 \mathrm{GHz}$. (b) $3.0 \mathrm{GHz}$. (c) $4.0 \mathrm{GHz}$.

\section{SIMULATION RESULTS}

The impedance bandwidth, radiation patterns, and the gain of the proposed dipole antenna have been simulated using CST microwave studio 2016. Fig. 3 plots the reflection coefficient $\mathrm{S}_{11}$ of the dipole antenna, and shows that the achieved impedance bandwidth $\left(\mathrm{S}_{11} \leq-10 \mathrm{~dB}\right)$ is $72.5 \%$ from 1.96 $\mathrm{GHz}$ to $4.19 \mathrm{GHz}$. Normalized radiation patterns of the dipole antenna in the E- and H-planes are displayed in Fig. 4. The realized pattern bandwidth is $66 \%$ from $1.96 \mathrm{GHz}$ to $3.90 \mathrm{GHz}$, within which the pattern maintains one main lobe fixed in the broadside direction in the E-plane, and a good omnidirectional property with a gain variation less than $0.3 \mathrm{~dB}$ in the H-plane. The cross-polarization levels across the entire bandwidth are below $-28 \mathrm{~dB}$. As shown in Fig. 5, the gain varies from $2.0 \mathrm{dBi}$ to $4.2 \mathrm{dBi}$ within the operating bandwidth.

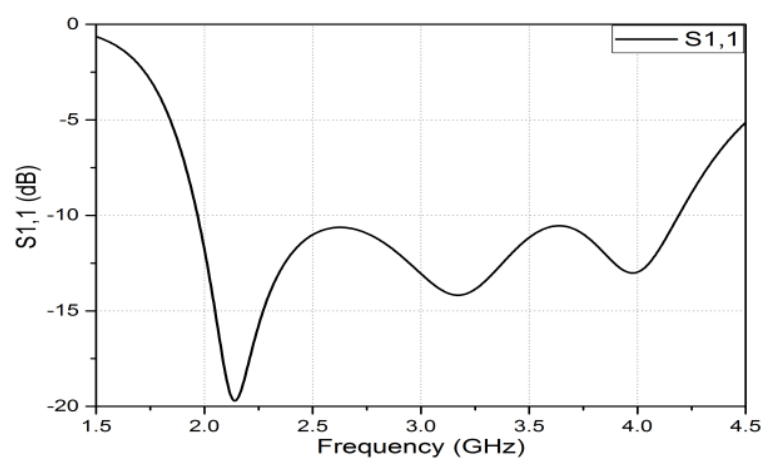

Fig. 3. Reflection coefficient values of the proposed antenna.

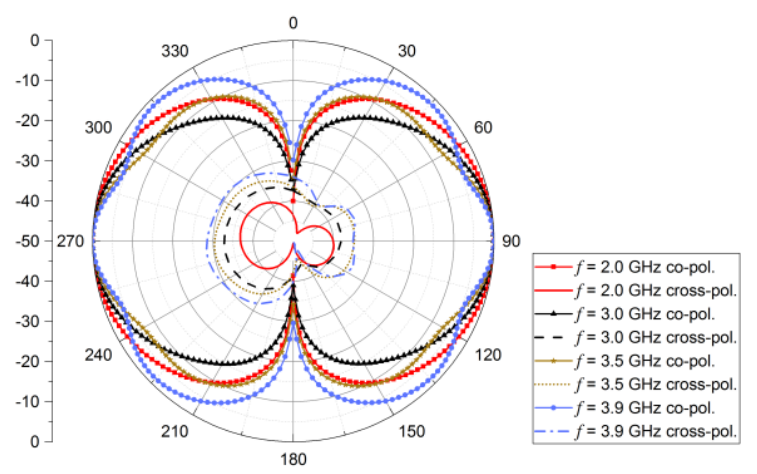

(a)

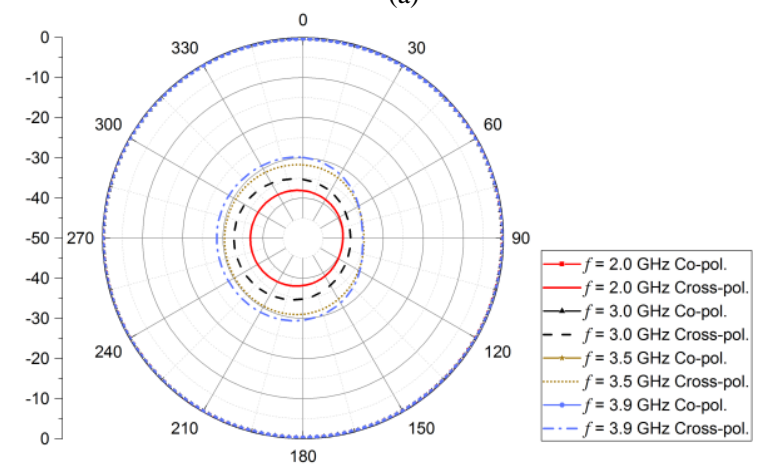

(b)

Fig. 4. Normalized radiation patterns of the proposed antenna. (a) E-plane. (b) H-plane. 


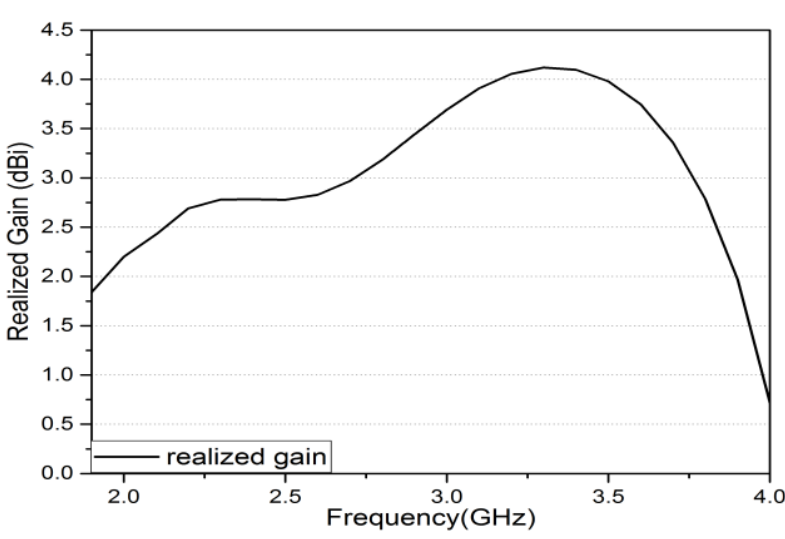

Fig. 5. Realized gain.

\section{CONCLUSION}

The design of a wideband omnidirectional dipole antenna, with its arms comprising of a non-uniformly segmented line structure, has been presented in this paper. The proposed structure mitigates the problem of excessive phase variation of currents flowing in it, which in turn helps the proposed antenna to maintain a stable omnidirectional pattern over a very wide band (1.96-3.90 GHz), accompanied by the enhanced gain performance.

\section{REFERENCES}

[1] J. D. Kraus and R. J. Marhefka, Antennas: For All Applications, 3rd ed. New York, NY, USA: McGraw-Hill, 2002.

[2] Z. Chen, D. Liu, H. Nakano, X. Qing, and T.Zwick, Handbook of Antenna Technologies, 1st ed. Singapore: Springer Singapore, 2016.

[3] S. Fan, Y. Yin, W. Hu, B. Li, and J. Yang, "Bandwidth enhancement of a printed dipole antenna for wideband applications," Microwave and Optical Technology Letters, vol. 54, pp. 1585-1590, 2012.

[4] Q. He, B. Wang, and J. He, "Wideband and dual-band design of a printed dipole antenna," IEEE Antennas and Wireless Propagation Letters, vol. 7, pp. 1-4, 2008.

[5] W. Yeoh, K. Wong, and W. Rowe, "Wideband miniaturized half bowtie printed dipole antenna with integrated balun for wireless applications," IEEE Transactions on Antennas and Propagation, vol. 59, pp. 339-342, 2011

[6] Z. Zhang, X. Yang, S. Zuo, and G. Fu, "Wideband omnidirectional printed dipole antenna with dumbbell-shaped open sleeve for wireless communication applications," IET Microwaves, Antennas \& Propagation, vol. 8, pp. 1299-1304, 2014.

[7] A. D. Yaghjian and S. R. Best, "Impedance, bandwidth, and Q of antennas," IEEE Trans. Antennas Propag., vol. 53, pp. 1298-1324, Apr.2005.

[8] D. M. Dobkin, S. M. Weigand, and N. Iyec, "Segmented magnetic antennas for near-field UHF RFID," Microw. J., vol. 50, no. 6, Jun. 2007.

[9] J. Shi, X. Qing, Z. N. Chen, and C. K. Goh, "Electrically large dualloop antenna for UHF near-field RFID reader," IEEE Transactions on Antennas and Propagation, vol. 61, pp. 1019-1025, 2013.

[10] X. Qing, C. Goh, and Z. Chen, "Segmented loop antenna for UHF near-field RFID applications," Electronics Letters, vol. 45, pp. 872873, 2009.

[11] K. Wei, Z. Zhang, Z. Feng, and M. F. Iskander, "A MNG-TL loop antenna array with horizontally polarized omnidirectional patterns," IEEE Transactions on Antennas and Propagation, vol. 60, pp. 27022710,2012
[12] K. Wei, Z. Zhang, Z. Feng, and M. F. Iskander, "A wideband MNGTL dipole antenna with stable radiation patterns," IEEE Transactions on Antennas and Propagation, vol. 61, pp. 2418-2424, 2013. 